\title{
Phytoconstituents of Adenanthera pavonina Linn from the bark extracts
}

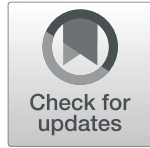

\author{
Arzumand Ara ${ }^{1}$, Md. Moshfekus Saleh-E-In ${ }^{1 *}$ (D, Md. Abul Hashem ${ }^{1}$, Mesbahuddin Ahmad ${ }^{1}$ and \\ Choudhury Mahmood Hasan ${ }^{2}$
}

\begin{abstract}
Background: Adenanthera pavonina L. is an important medicinal plant and its barks are used in traditional medicine for treating different diseases. Therefore, a phytochemical investigation was carried out to isolate and identify secondary metabolites from its barks.

Results: Seven compounds namely ethyl 3,3-dimethyl-13-hydroxytridecanoate (1), stigmasta-5,22-dien-3ß-ol (2), tert.butyl tridecanoate (3), 6-a-hydroxy stigmast-20(21)-en-3-one (4) of dichloromethane extract and 18-(2', 3'dihydroxyphenyl)nonadec-17-en-2-ol (5), 1-(N-propyl amino)-2-henecosanone (6), and stigmast-5(6), 20(21)-diene-3one (7) were isolated from the barks of Adenanthera pavonina Linn. Of these compounds, 1, 4, 5, 6, and 7 appear new. The structures of these compounds were elucidated by spectroscopic techniques, mainly by NMR.

Conclusions: Five new and two known compounds have been isolated and characterized from the bark of $A$. pavonina. The isolated compounds could be a potential template for the synthesis and development of new lead compounds with interesting pharmacological properties.
\end{abstract}

Keywords: Adenanthera pavonina, Bark, Dichloromethane extract, Ethyl acetate extract, Chromatography

\section{Background}

Adenanthera pavonina L (Bengali: Rakta kambal) is an erect medium-sized tree $(6-15 \mathrm{~m}$ tall and up to $45-\mathrm{cm}$ diameter) with dark brown to grayish bark belongs to the family Leguminosae. The plant is native to the Asian continent and mostly found in Africa, Pacific and Caribbean regions [1]. It is also indigenous to India and Bangladesh particularly in the South-eastern region [2]. Different parts of this plant have been used in traditional medicine for the treatment of various diseases. The bark and leaves are used as a remedy for diarrhea, gout, hematuria, hematemesis, and chronic rheumatism [1-5]. The anti-inflammatory, analgesic, antioxidant, cytotoxic, anti-diarrheal, acute toxicity, antibacterial, antifungal, and blood pressure-reducing activities of the bark, leaf, and seed extracts and its isolated compounds have been reported [6-13]. Previous phytochemical investigation reported the presence of many bioactive compounds like robinetin, chalcone, butin and flavanol ampelopsin,

\footnotetext{
* Correspondence: saleheen.sosthe@gmail.com

${ }^{1}$ Department of Chemistry, Jahangirnagar University, Savar, Dhaka 1342,

Bangladesh

Full list of author information is available at the end of the article
}

stigmasterol glucosides, oleanolic acid, echinocystic acid, and sapogenins from the leaves and seeds of the plant $[6$, 14-19]. Very few compounds like as stigmasterol glucosides, oleanolic acid, and echinocystic acid have been reported from the bark part $[15,20]$. Our previous in vitro studies have shown that the bark extracts of the plant possessed significant pharmacological activities [11-13]. Hence, our aim is to isolate bioactive compounds from this plant part.

\section{Methods}

\subsection{Chemicals}

n-Hexane, dichloromethane, chloroform, ethyl acetate, and methanol (Merck Germany) were used for solvent extraction. The laboratory grade petroleum ether (bp. $40-60{ }^{\circ} \mathrm{C}$ ) was collected from fuel petrol by fractional distillation. Silica gel $60 \mathrm{H}$ (E Merck, 7731), silica gel $60(0.063-0.200 \mathrm{~mm})$, vanillin, and sulfuric acid were from Merck, Germany.

\subsection{General experimental procedures}

Melting points were recorded by using an electro-thermal melting point apparatus (Stuart Scientific SMP3, UK) and OptiMelt (MPA100, Stanford Research Systems, USA). IR 
spectra were recorded using Nicolet iS10 FT-IR spectrometer by potassium bromide $(\mathrm{KBr})$ pellets. ${ }^{1} \mathrm{H}$ NMR, ${ }^{13} \mathrm{C}$ NMR, DEPT 135 spectra, and attached proton test (APT) spectra were recorded in $\mathrm{CDCl}_{3}, \mathrm{CD}_{3} \mathrm{OD}$, and mixture of $\mathrm{CDCl}_{3}$ and $\mathrm{CD}_{3} \mathrm{OD}$ with a $300-\mathrm{MHz}$ NMR Spectrometer (Varian MERCURY-VX-300). Chemical shifts are presented in $\delta$ (ppm) using tetramethylsilane (TMS) as an internal standard, and coupling constants $(()$ are expressed in Hertz $(\mathrm{Hz})$. Mass spectra were recorded by infusion into the ESI source using $\mathrm{CH}_{3} \mathrm{OH} / \mathrm{CH}_{3} \mathrm{OD}$ as a solvent with a LC-ESI-MS/MS-System TSQ Quantum Ultra AM Finnigan and Triple Quadrupole MS with Mikro-HPLC (Surveyor plus). Pre-coated glass plates of silica gel (Keiselgel 60, F254, Merck KGaA, Darmstadt, Germany) were used for TLC analysis. The TLC spots were observed under long and short wavelength UV light (Fisher Scientific LCF-445) at 366 and $254 \mathrm{~nm}$ and the plates were sprayed with vanillin-sulfuric acid solution.

\subsection{Plant material}

The barks of Adenanthera pavonina were collected from the capital city Dhaka of Bangladesh. The plant was authenticated by Dr. Sardar Nasir Uddin of Bangladesh National Herbarium, Dhaka, and a voucher specimen (accession number-34196) was deposited in the Herbarium.

\subsection{Extraction and isolation}

The barks were air dried and ground into a powder. The powder $(2.25 \mathrm{~kg})$ was extracted successively with petroleum ether (b.p. $\left.40-60^{\circ} \mathrm{C}\right)(3 \times 4 \mathrm{~L})$, dichloromethane $(3 \times$ $2.5 \mathrm{~L})$, ethyl acetate $(3 \times 2.5 \mathrm{~L})$, and methanol $(3 \times 2.5 \mathrm{~L})$ at room temperature for $72 \mathrm{~h}$ of each. The extracts were then concentrated in vacuo by rotary evaporator (Bucho, R$15 \mathrm{v})$. A yellowish brown (4.42 g), greenish brown $(8.0 \mathrm{~g})$, reddish brown $(5.7 \mathrm{~g})$, and maroon $(65.1 \mathrm{~g})$ color extracts were obtained from the petroleum ether, dichloromethane, ethylacetate, and methanol extracts, respectively. Based on previous pharmacological investigation [11, 12], the crude dichloromethane (DCM) extract was selected for isolation of compounds. Therefore, the dichloromethane extract was run on TLC before extensive chromatographic separation, the solvent system that gave the best resolution in EtOAc:Petroleum ether (1:9). Seven spots $\left(\mathrm{R}_{f}\right.$ $0.93,0.89,0.84,0.68,0.48,0.34$, and 0.18 ) were observed with tailing. After TLC study, the extract was loaded on a vacuum liquid chromatograhy (VLC) and the column was packed with silica gel $(60 \mathrm{H})$. The column was eluted successively (according to their polarity index) with hexane, dichloromethane, ethyl acetate, and methanol by different polarity ratios at $200 \mathrm{~mL}$ of each. A total of 10 fractions were obtained. The fraction $1(49 \mathrm{mg})$ and fraction $2(6$ $\mathrm{mg}$ ) gave the same $\mathrm{R}_{f}$ value (0.8) in $100 \%$ petroleum ether (pet.ether). Therefore, fraction 1 and 2 were pooled together and obtained a pure brown needle-shaped crystal
(18 mg, m.p. $115^{0}$ C, $\mathrm{R}_{f} 0.8$ ) of compound $\mathbf{1}$ (ethyl 3,3-dimethyl-13-hydroxytridecanoate) by re-crystallization method with hot methanol. Fraction $6(1.49 \mathrm{~g})$ eluted successively with pet.ether, DCM, EtOAc, and $\mathrm{MeOH}$ by different solvent ratio through glass chromatographic column $(90 \mathrm{~cm} \times 8 \mathrm{~cm}$, internal diameter $)$ to obtain 10 fractions (6A to $6 \mathrm{~J})$. Fraction $6 \mathrm{E}(220 \mathrm{mg})$ was further eluted with $10 \%$ dichloromethane in petroleum ether to give $22 \mathrm{mg}$ of compound 2 (stigmasta-5,22-dien-3 $\beta$-ol). The eighth fraction, $6 \mathrm{H}(90 \mathrm{mg})$ was subjected to again column chromatography (CC) over silica gel 60 and eluted with mixtures of EtOAc: pet.ether (1:9) which furnished 6 mg of compound 3 (tert.butyl tridecanoate). Fraction 6I $(170 \mathrm{mg})$ was then separated by $10 \%$ pet.ether in chloroform by preparative thin layer chromatography (PTLC) method and yielded $10 \mathrm{mg}$ of compound 4 (6- $\alpha$-hydroxy stigmast-20(21)-en-3-one).

According to the previous biological studies on the different extracts of the barks [11, 12], the ethyl acetate extract had been considered for further chromatographic separation, The crude extract was checked on TLC before undergoing extensive chromatographic separation. The best resolution was observed in EtOAc:pet.ether (1:9) and found seven distinct spots with tailing. Then, the EtOAc extract $(3.1 \mathrm{~g})$ was started for separation by column chromatography by using pet-ether-DCM, DCM, DCM-EtOAc, EtOAc, and $\mathrm{MeOH}$ as solvent systems according to their polarity. This procedure gave 6 fractions. The fractions $1(10 \mathrm{mg})$ and $3(7 \mathrm{mg})$ checked on TLC with 100\% pet.ether and pet.ether-DCM (9: $1)$ and were obtained as pure compounds and labeled as compounds 5 (18-(2', 3'-dihydroxyphenyl)nonadec-17-en2-ol) and 6 (1-(N-propyl amino)-2-henecosanone), respectively. The fraction 4 of EtOAc extract $(26 \mathrm{mg})$ was further eluted with pet-ether-DCM and DCM solvent systems by column chromatography and yielded two sub-fraction 4A (6 $\mathrm{mg})$ and $4 \mathrm{~B}(14 \mathrm{mg})$. The fraction $4 \mathrm{~B}$ was further purified by PTLC method (solvent system, pet.ether: $\mathrm{CHCl}_{3}=4: 1$ ) which resulted in the isolation $3 \mathrm{mg}$ of compound 7 (stigmast-5(6), 20(21)-diene-3-one).

\section{Results}

\subsection{Characteristic data of compounds}

\subsubsection{Ethyl 3,3-dimethyl-13-hydroxytridecanoate (1)}

Brown needle-shaped crystal $(18 \mathrm{mg})$. Soluble in pet.ether. mp. $115^{\circ} \mathrm{C} . \mathrm{R}_{f}=0.80$ (100\% pet.ether). ${ }^{1} \mathrm{H}$ NMR $\left(\mathrm{CDCl}_{3}\right.$ $400 \mathrm{MHz}): \delta 4.05(\mathrm{q}, J=7.17 \mathrm{~Hz}, 2 \mathrm{H}, \mathrm{H}-14), 3.33(\mathrm{dt}, 2 \mathrm{H}$, H-13), 1.98 (s, 2H, H-2), 1.54 (m, 2H, H-12), 1.2-1.34 (m, $16 \mathrm{H}, \mathrm{H}-4,5,6,7,8,9,10,11), 0.81$ (t, 3H, H-15), 0.79 (s, 3H, H16, 17), and 1.62 (br s, $1 \mathrm{H}, 13-\mathrm{OH})$. ESI-MS (Negative ion): $\mathrm{m} / \mathrm{z} 283.7\left(\mathrm{M}^{+}-2 \mathrm{H}\right)$. Calculated for $\mathrm{C}_{17} \mathrm{H}_{34} \mathrm{O}_{3}=286.2508$.

\subsubsection{Stigmasta-5,22-dien-3 $\beta$-ol (2)}

White needle-shaped crystal $(22 \mathrm{mg})$. Soluble in $\mathrm{CHCl}_{3}$, mp $164{ }^{\circ} \mathrm{C} . \mathrm{R}_{f}=0.53$ (pet.Ether: $\left.\mathrm{CH}_{2} \mathrm{Cl}_{2}=1: 19\right)$. IR (KBr): 
3676 (br,-OH str), 2960, 2852 (C-H str), 1558, 1601 (C$\mathrm{H}$ bend), 1051, 960, $800 \mathrm{~cm}^{-1} \cdot{ }^{1} \mathrm{H}$ NMR $\left(\mathrm{CDCl}_{3} 400\right.$ $\mathrm{MHz}): \delta 5.35(\mathrm{~d}, 1 \mathrm{H}, \mathrm{H}-6), 5.15(\mathrm{dd}, 1 \mathrm{H}, J=8.52$ and $15.02 \mathrm{~Hz}, \mathrm{H}-22), 5.03$ (dd, $1 \mathrm{H}, J=8.52$ and $15.02 \mathrm{~Hz}, \mathrm{H}-$ 23), $3.51(\mathrm{~m}, 1 \mathrm{H}, \mathrm{H}-3), 2.27(\mathrm{~m}, 1 \mathrm{H}, \mathrm{H}-20), 2.23(\mathrm{~m}, 1 \mathrm{H}$, $\mathrm{H}-24), 2.22$ (m, 2H, H-4), 1.85 (m, $2 \times 1 \mathrm{H}, \mathrm{H}-25,7), 1.54$ $(\mathrm{m}, 1 \mathrm{H}), 1.53(\mathrm{~m}, 3 \times 2 \mathrm{H}, \mathrm{H}-15,16,17), 1.44(\mathrm{~m}, 3 \times 1 \mathrm{H}$, $6 \times 2 \mathrm{H}, \mathrm{H}-2,8,9,11,12,14), 1.25(\mathrm{~m}, 2 \times 2 \mathrm{H}, \mathrm{H}-1,28)$, 1.08 (d, 3H, H-21), 1.00 (s, 3H, H-19), 0.84 (d, 3H, J= $6.12 \mathrm{~Hz}, \mathrm{H}-27), 0.82(\mathrm{t}, 3 \mathrm{H}, J=6.98 \mathrm{~Hz}, \mathrm{H}-29), 0.69$ (s, $3 \mathrm{H}, \mathrm{H}-18$ ), 0.68 (d, $3 \mathrm{H}, J=6.07 \mathrm{~Hz}, \mathrm{H}-26) .{ }^{13} \mathrm{C}$ NMR $\left(\mathrm{CDCl}_{3}, 100 \mathrm{MHz}\right): \delta 140.79(\mathrm{C}-5), 138.32(\mathrm{C}-22), 129.33$ (C-23), 121.72 (C-6), 71.83 (C-3), 56.91 (C-14), 56.02 (C17), 51.27 (C-24), 50.21 (C-9), 42.35 (C-13), 42.26 (C-4), 40.48 (C-20), 39.73 (C-12), 37.30 (C-1), 36.55 (C-10), 31.94 (C-25,8), 31.71 (C-7,2), 28.93 (C-16), 25.42 (C-28), 24.39 (C-15), 21.23 (C-26,11), 21.10 (C-21), 19.41 (C-19), 19.01 (C-27), 12.25 (C-29), 12.07 (C-18). ESI-MS (positive ion): at $\mathrm{m} / \mathrm{z} 413.6\left(\mathrm{M}^{+}+\mathrm{H}\right)$. Calculated for $\mathrm{C}_{29} \mathrm{H}_{48} \mathrm{O}=412.3705$ [21].

\subsection{3 tert.butyl tridecanoate (3)}

White solid (6 mg). Soluble in $\mathrm{CHCl}_{3} . \mathrm{mp} 75^{\circ} \mathrm{C} . \mathrm{R}_{f} 0.77$ in 100\% DCM. IR (KBr):1707 (C=Ostrc), 2919 and 2843 (aliphatic $\mathrm{C}-\mathrm{H}$ strc), 1464 and 1377 (aliphatic $\mathrm{C}-\mathrm{H}$ bending), 1194 and 1096 (C-O strc) $\mathrm{cm}^{-1}$. ${ }^{1} \mathrm{H}$ NMR $\left(\mathrm{CDCl}_{3} 400\right.$ $\mathrm{MHz}): \delta 2.34(\mathrm{t}, 2 \mathrm{H}, J=7.40 \mathrm{~Hz}, \mathrm{H}-2), 1.62(\mathrm{~m}, 2 \mathrm{H}, \mathrm{H}-3)$, $1.60(\mathrm{~m}, 2 \mathrm{H}, \mathrm{H}-4), 1.25(\mathrm{~s}, 3 \times 3 \mathrm{H}, \mathrm{H}-15,16,17), 1.24(\mathrm{~m}$, $8 \times 2 \mathrm{H}, \mathrm{H}-5,6,7,8,9,10,11,12), 0.87(\mathrm{t}, 3 \mathrm{H}, J=6.80 \mathrm{~Hz}, \mathrm{H}-$ 13). ${ }^{13} \mathrm{C}$ NMR $\left(\mathrm{CDCl}_{3}, 100 \mathrm{MHz}\right): \delta 178.30(\mathrm{C}-1), 76.72(\mathrm{C}-$ 14), 33.78 (C-2), 31.96 (C-3), 29.73 (C-4, 5), 29.47 (C-6, 7), 29.39 (C-15, 16, 17), 29.27 (C-8,9), 29.10 (C-10), 24.74 (C11), 22.72 (C-12), 14.13 (C-13). ESI-MS (positive ion): at $\mathrm{m} /$ z $271.3\left(\mathrm{M}^{+}+\mathrm{H}\right)$. Calculated for $\mathrm{C}_{17} \mathrm{H}_{34} \mathrm{O}_{2}=270.2559$. On the basis of these spectral data, compound 3 was identified as tert.butyl tridecanoate (http://www.nmrdb.org) (Fig. 1).

\subsubsection{6-a-hydroxy stigmast-20(21)-en-3-one (4)}

Brown semi-solid $(10 \mathrm{mg})$. Soluble in $\mathrm{CHCl}_{3} . \mathrm{R}_{f}=0.52$ (pet.ether: $\left.\mathrm{CHCl}_{3}=1: 95\right)$. IR ( $\left.\mathrm{KBr}\right)$ : 3450 (O-H str), 2940, 2869 ( $\mathrm{C}-\mathrm{H}$ str), 1686 ( $\mathrm{C}=\mathrm{O}$ str), 1641 ( $\mathrm{C}=\mathrm{C}$ str), 1034 (C-O str), 983, and $883 \mathrm{~cm}^{-1} \cdot{ }^{1} \mathrm{H}$ NMR $\left(\mathrm{CDCl}_{3}\right.$ $400 \mathrm{MHz}$ ): $\delta .4 .73$ (br, s, 1H, H-21b), 4.60 (br, s, 1H, H21a), 4.15 (distorted $t, 1 \mathrm{H}, \mathrm{H}-6$ ), 3.65 (distorted $\mathrm{t}, 2 \mathrm{H}$, $\mathrm{H}-2$ ), 3.18 (br, 2H, H-4), 2.99 (distorted t, 2H, H-1), 2.71 (m, 1H, H-17), 2.27 (m, 1H, H-5), 2.18 (m, 1H, H-24), $1.95(\mathrm{~m}, 2 \mathrm{H}, \mathrm{H}-11), 1.67(\mathrm{~m}, 2 \mathrm{H}, \mathrm{H}-22), 1.60(\mathrm{~m}, 1 \mathrm{H}, \mathrm{H}-$ 25), $1.52(\mathrm{~m}, 2 \times 2 \mathrm{H}, \mathrm{H}-15,16), 1.37$ (m, $2 \mathrm{H}, \mathrm{H}-28), 1.24-$ $1.36(\mathrm{~m}, 2 \times 2 \mathrm{H}, 2 \times 1 \mathrm{H}, \mathrm{H}-12,7,8,9), 1.27(\mathrm{~m}, 2 \mathrm{H}, \mathrm{H}-23)$, $1.24(\mathrm{~m}, 1 \mathrm{H}, \mathrm{H}-14), 0.96$ (d, $3 \mathrm{H}, J=7.0 \mathrm{~Hz}, \mathrm{H}-26), 0.94$ (d, 3H, $J=7.0 \mathrm{~Hz}, \mathrm{H}-27$ ), 0.87 (distorted t, 3H, H-29), 0.81 (s, 3H, H-18), 0.74 (s, 3H, H-19). ${ }^{13} \mathrm{C}$ NMR $\left(\mathrm{CDCl}_{3}\right.$, $100 \mathrm{MHz}): \delta 180.3$ (C-3), 150.4 (C-20), 109.7 (C-21), 79.1 (C-6), 56.3 (C-13), 56.0 (C-17), 55.4 (C-9), 46.9 (C-
4), 42.5 (C-14), 40.5 (C-22), 38.9 (C-10), 37.3 (C-5,8), 37.0 (C-1), 34.4 (C-2, 23), 32.2 (C-12), 31.9 (C-7), 29.4 (C16), 27.4 (C-24), 24.9 (C-15, 28), 22.7 (C-11), 20.9 (C-27), 19.4 (C-26), 14.1 (C-29), 18.3 (C-18), 14.7 (C-19). On the basis of these spectral data, compound 4 was identified as 6- $\alpha$-hydroxy stigmast-20(21)-en-3-one (Fig. 1).

\subsubsection{8-(2', 3'-dihydroxyphenyl)nonadec-17-en-2-ol (5)}

White crystal $(10 \mathrm{mg})$. Soluble in $\mathrm{CHCl}_{3}, \mathrm{mp}$. 106$108^{\circ} \mathrm{C} . \mathrm{R}_{f}=0.85$ (pet.ether). IR ( $\left.\mathrm{KBr}\right): 3425$ (O-H str), 2924 and 2852 (C-H aliphatic str.), 1558 and $1543(\mathrm{C}=\mathrm{C}$, aliphatic), $1506\left(\mathrm{C}=\mathrm{C}\right.$, aromatic) $\mathrm{cm}^{-1} \cdot{ }^{1} \mathrm{H}$ NMR $\left(\mathrm{CDCl}_{3}\right.$ $400 \mathrm{MHz}): \delta 8.08\left(\mathrm{~d}, 1 \mathrm{H}, J=8.0 \mathrm{~Hz}, \mathrm{H}-4^{\prime}\right), 7.60(\mathrm{~d}, 1 \mathrm{H}$, $J=7.2 \mathrm{~Hz}, \mathrm{H}-6^{\prime}$ ), 7.47 (dd, $1 \mathrm{H}, J=8.0$ and $7.2 \mathrm{~Hz}, \mathrm{H}-5^{\prime}$ ), 5.42 (distorted triplet, $1 \mathrm{H}, \mathrm{H}-17), 3.9(\mathrm{~m}, 1 \mathrm{H}, \mathrm{H}-2), 2.35$ (distorted triplet, 2H, H-16), 1.24-1.53 (m, 13×2H, H-315), 2.03 (s, 3H, H-19), 0.86 (d, 3H, $J=7.92 \mathrm{~Hz}, \mathrm{H}-1$ ). ESI-MS (positive ion) $\mathrm{m} / \mathrm{z} 413.2\left(\mathrm{M}^{+}+\mathrm{Na}\right)$ and GC-MS $\mathrm{m} / \mathrm{z} 391\left(\mathrm{M}^{+}+\mathrm{H}\right)$.

\subsubsection{1-(N-propyl amino)-2-henecosanone (6)}

White waxy solid $(7 \mathrm{mg})$. Soluble in $\mathrm{CHCl}_{3} . \mathrm{mp} 67^{\circ} \mathrm{C} . \mathrm{R}_{f}$ 0.58 (in pet.ether: $\mathrm{DCM}=9: 1)$. IR $(\mathrm{KBr}): 2916$ and 2848 (aliphatic C-H str.), 1701 (C=O str) $\mathrm{cm}^{-1} \cdot{ }^{1} \mathrm{H}$ NMR $\left(\mathrm{CDCl}_{3} 400 \mathrm{MHz}\right): \delta 4.04(\mathrm{~s}, \mathrm{br}, 2 \mathrm{H}, \mathrm{H}-1), 2.34(\mathrm{t}, 2 \mathrm{H}, J=$ $\left.7.4 \mathrm{~Hz}, \mathrm{H}-3,1^{\prime}\right), 1.63$ (m, 2H, H-2'), 1.24 (m, 34H, H-4 to 20), $0.86\left(\mathrm{t}, 3 \mathrm{H}, J=6.28 \mathrm{~Hz}, \mathrm{H}-21,3^{\prime}\right), 2.16(\mathrm{br}, \mathrm{N}-\mathrm{H}) .{ }^{13} \mathrm{C}$ NMR $\left(\mathrm{CDCl}_{3}, 100 \mathrm{MHz}\right): \delta 204(\mathrm{C}-2), 67.5$ (C-1), 57.6 (C$\left.1^{\prime}\right)$, 33.65 (C-3), 33.65, 31.95, 29.45, 29.37, 29.09, 24.43, 22.71 (C-4 to 20), 24.43 (C-2'),14.13 (C-21, 3').

\subsubsection{Stigmast-5(6), 20(21)-diene-3-one (7)}

Brown semi-solid (3 mg). Soluble in $\mathrm{CHCl}_{3} . \mathrm{R}_{f}=0.51$ (pet.ether: $\left.\mathrm{CHCl}_{3}=1: 4\right) .{ }^{1} \mathrm{H}$ NMR $\left(\mathrm{CDCl}_{3} 400 \mathrm{MHz}\right): \delta$ 5.35 (distorted triplet, 1H, H-6), 4.72 (br, s, $1 \mathrm{H}, \mathrm{H}-21 \mathrm{a}$ ), 4.59 (br, s, 1H, H-21b), 3.65 (s,2H, H-4), 3.17 (distorted triplet, $2 \mathrm{H}, \mathrm{H}-2), 2.99$ (distorted triplet, $2 \mathrm{H}, \mathrm{H}-1), 2.98$ (m, $1 \mathrm{H}, \mathrm{H}-17), 2.27$ (m, 1H, H-24), 1.68 (m, 2H, H-7), 1.63 (m, 1H, H-25), 1.60 (distorted triplet, 2H, H-22), 1.51 (m, $2 \times 2 \mathrm{H}, \mathrm{H}-15,16), 1.36(\mathrm{~m}, 2 \times 2 \mathrm{H}, \mathrm{H}-23,28), 1.24(\mathrm{~m}$, $3 \times 1 \mathrm{H}, 2 \times 2 \mathrm{H}, \mathrm{H}-8,9,11,12,14), 0.96(\mathrm{~d}, 3 \mathrm{H}, J=6.8 \mathrm{~Hz}$, $\mathrm{H}-26), 0.94$ (d, $3 \mathrm{H}, J=6.8 \mathrm{~Hz}, \mathrm{H}-27), 0.86(\mathrm{t}, 3 \mathrm{H}, J=6.9$ Hz, H-29), 0.81 (s, 3H, H-19), 0.74 (s, 3H, H-18).

\section{Discussion}

\subsection{Characterization of compounds}

\subsubsection{Compound 1}

The ${ }^{1} \mathrm{H}-\mathrm{NMR}$ data (Table 1 ) of compound $\mathbf{1}$ showed a triplet at $\delta 0.81(\mathrm{H}-15)$ and a quartet at $\delta 4.05(J=7.17 \mathrm{~Hz}, \mathrm{H}-$ 14). It indicates the presence of $\mathrm{O}-\mathrm{CH}_{2}-\mathrm{CH}_{3}$ group. The peak at $\delta 1.62(\mathrm{br}, \mathrm{s},-\mathrm{OH})$ and a distorted triplet at $\delta 3.33$ $(\mathrm{H}-13)$ is responsible for a primary hydroxyl group. The long chain of this compound is confirmed by a multiplet at $\delta$ 1.20-1.34 whose intensity indicates eight $\mathrm{CH}_{2}$ group. A 


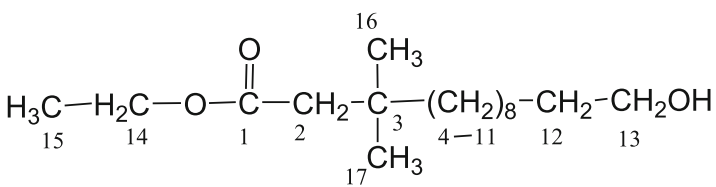

Ethyl 3,3-dimethyl-13-hydroxy tridecanoate (1)<smiles>CCCCCC(C)(C)OC(=O)CC</smiles>

Tert butyl tridecanoate (3)<smiles>C/C(=C/CCC(C)O)c1cccc(O)c1O</smiles>

18-(2', 3' -Dihydroxyphenyl)nonadec-17-en-2-ol (5)<smiles>CC=CCC(=O)CCNCCC</smiles>

1-(N-propyl amino)-2-henecosanone (6)<smiles>CC1CCC2C3CC=C4CC(=O)CCC4(C)C3CCC12C</smiles>

Sigmast-5(6), 20(21)-diene-3-one (7)

Fig. 1 Isolated compounds from the bark of Adenanthera pavonina Linn.

tertiary carbon substituted by two methyl groups into the chain which gave a peak at $\delta 0.79$ (s, H-16,17). The ESI-MS spectrum showed peak at $\mathrm{m} / \mathrm{z} 283.7$ in the negative ion

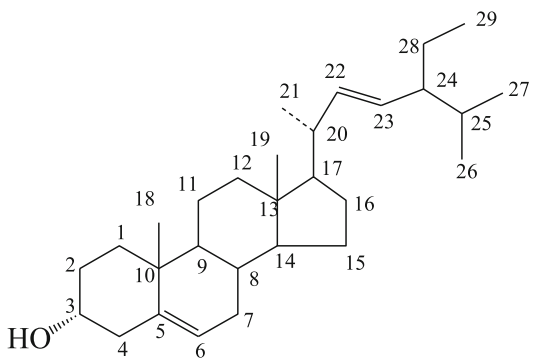

Stigmasta-5,22-dien-3 -ol (2)<smiles>CCC(/C=C\CC(CC)C1CCC2C3CC(O)C4CC(=O)CCC4(C)C3CCC2(C)C1C)C(C)C</smiles>

6- $\alpha$-hydroxy stigmast-20(21)-en-3-one (4) 
Table $1{ }^{1} \mathrm{H}$ and ${ }^{13} \mathrm{C}$ NMR $\delta$ (ppm) data of compounds 1, 4-7

\begin{tabular}{|c|c|c|c|c|c|c|c|}
\hline \multirow[t]{2}{*}{ Carbon no. } & \multirow{2}{*}{$\begin{array}{l}1 \\
{ }^{1} \mathrm{H} \delta(\mathrm{ppm})\end{array}$} & \multicolumn{2}{|l|}{4} & \multirow{2}{*}{$\begin{array}{l}5 \\
{ }^{1} \mathrm{H} \delta(\mathrm{ppm})\end{array}$} & \multicolumn{2}{|l|}{6} & \multirow{2}{*}{$\begin{array}{l}7 \\
{ }^{1} \mathrm{H} \delta(\mathrm{ppm})\end{array}$} \\
\hline & & $\overline{{ }^{1} \mathrm{H} \delta(\mathrm{ppm})}$ & ${ }^{13} \mathrm{C} \delta(\mathrm{ppm})$ & & ${ }^{1} \mathrm{H} \delta(\mathrm{ppm})$ & ${ }^{13} \mathrm{C} \delta(\mathrm{ppm})$ & \\
\hline 1 & - & $\begin{array}{l}2.99 \\
\text { (distorted t, 2H) }\end{array}$ & 37.0 & $\begin{array}{l}0.86 \\
(\mathrm{~d}, 3 \mathrm{H}, J=7.92 \mathrm{~Hz})\end{array}$ & $4.04(\mathrm{~s}, \mathrm{br}, 2 \mathrm{H})$ & 67.5 & $\begin{array}{l}2.99 \\
\text { (distorted triplet,2H) }\end{array}$ \\
\hline 2 & $1.98(\mathrm{~s}, 2 \mathrm{H})$ & \multirow[t]{2}{*}{$\begin{array}{l}3.65 \\
\text { (distorted t, 2H) }\end{array}$} & 34.4 & $3.87(\mathrm{~m}, 1 \mathrm{H})$ & & 204 & $\begin{array}{l}3.17 \\
\text { (distorted triplet, } 2 \mathrm{H}, \mathrm{H}-2 \text { ) }\end{array}$ \\
\hline 3 & & & 180.3 & $1.24-1.53(\mathrm{~m}, 13 \times 2 \mathrm{H})$ & $\begin{array}{l}2.34 \\
(\mathrm{t}, 2 \mathrm{H}, J=7.4 \mathrm{~Hz})\end{array}$ & 33.65 & \\
\hline 4 & \multirow{8}{*}{$\begin{array}{l}1.2-1.34 \\
(\mathrm{~m}, 16 \mathrm{H})\end{array}$} & $3.18(\mathrm{br}, 2 \mathrm{H})$ & 46.9 & & $1.24(\mathrm{~m}, 34 \mathrm{H})$ & 22.71 & $3.65(\mathrm{~s}, 2 \mathrm{H})$ \\
\hline 5 & & $2.27(\mathrm{~m}, 1 \mathrm{H})$ & 37.3 & & & $\begin{array}{l}24.43 \\
29.09\end{array}$ & \\
\hline 6 & & $4.15(\mathrm{~m}, 1 \mathrm{H})$ & 79.1 & & & $\begin{array}{l}29.37 \\
29.45\end{array}$ & 5.35 (distorted triplet, $1 \mathrm{H}$ ) \\
\hline 7 & & \multirow{2}{*}{$\begin{array}{l}1.24-1.36 \\
(\mathrm{~m}, 2 \times 2 \mathrm{H}, 1 \mathrm{H})\end{array}$} & 31.9 & & & 31.95 & \\
\hline 8 & & & 37.3 & & & & $1.68(\mathrm{~m}, 2 \mathrm{H})$ \\
\hline 9 & & & 55.4 & & & & $1.24(\mathrm{~m}, 1 \mathrm{H})$ \\
\hline 10 & & & 38.9 & & & & \\
\hline 11 & & $1.95(\mathrm{~m}, 2 \mathrm{H})$ & 22.7 & & & & $1.24(\mathrm{~m}, 2 \mathrm{H})$ \\
\hline 12 & $1.54(\mathrm{~m}, 2 \mathrm{H})$ & $1.24(\mathrm{~m}, 2 \mathrm{H})$ & 32.2 & & & & $1.24(\mathrm{~m}, 2 \mathrm{H})$ \\
\hline 13 & $\begin{array}{l}3.33 \\
\text { (distorted triplet, } 2 \mathrm{H}) \\
1.62(\mathrm{~s}, \mathrm{br}, \mathrm{OH})\end{array}$ & & 56.3 & & & & \\
\hline 14 & $4.05(q, J=7.17 \mathrm{~Hz}, 2 \mathrm{H})$ & $1.24(\mathrm{~m}, 1 \mathrm{H})$ & 42.5 & & & & $1.24(\mathrm{~m}, 1 \mathrm{H})$ \\
\hline 15 & $0.81(\mathrm{t}, 3 \mathrm{H})$ & $1.52(\mathrm{~m}, 2 \mathrm{H})$ & 24.9 & & & & $1.51(\mathrm{~m}, 2 \mathrm{H})$ \\
\hline 16 & $0.79(\mathrm{~s}, 3 \mathrm{H})$ & $1.52(\mathrm{~m}, 2 \mathrm{H})$ & 29.4 & $\begin{array}{l}2.35 \\
\text { (distorted triplet, } 2 \mathrm{H} \text { ) }\end{array}$ & & & $1.51(\mathrm{~m}, 2 \mathrm{H})$ \\
\hline 17 & $0.79(\mathrm{~s}, 3 \mathrm{H})$ & $2.71(\mathrm{~m}, 1 \mathrm{H})$ & 56.0 & $\begin{array}{l}5.42 \\
\text { (distorted triplet, } 1 \mathrm{H} \text { ) }\end{array}$ & & & $2.98(\mathrm{~m}, 1 \mathrm{H})$ \\
\hline 18 & & $0.81(\mathrm{~s}, 3 \mathrm{H})$ & 18.3 & & & & $0.74(\mathrm{~s}, 3 \mathrm{H})$ \\
\hline 19 & & $0.74(\mathrm{~s}, 3 \mathrm{H})$ & 14.7 & $2.03(\mathrm{~s}, 3 \mathrm{H})$ & & & $0.81(\mathrm{~s}, 3 \mathrm{H})$ \\
\hline 20 & & & 150.4 & & & & \\
\hline \multirow[t]{2}{*}{21} & & $\begin{array}{ll}\text { a } & 4.60 \\
& \text { (br, s, 1H) }\end{array}$ & 109.7 & & $\begin{array}{l}0.86 \\
(\mathrm{t}, 3 \mathrm{H}, J=6.28 \mathrm{~Hz})\end{array}$ & 14.13 & $4.72\left(\mathrm{br}, \mathrm{s}, 1 \mathrm{H}_{\mathrm{H}}\right)$ \\
\hline & & $\begin{array}{ll}\mathrm{b} & 4.73 \\
& \text { (br, s, 1H) }\end{array}$ & & & & & $4.59(\mathrm{br}, \mathrm{s}, 1 \mathrm{H})$ \\
\hline 22 & & $1.67(\mathrm{~m}, 2 \mathrm{H})$ & 40.5 & & & & $\begin{array}{l}1.60 \\
\text { (distorted triplet, } 2 \mathrm{H} \text { ) }\end{array}$ \\
\hline 23 & & $1.27(\mathrm{~m}, 2 \mathrm{H})$ & 27.4 & & & & $1.36(\mathrm{~m}, 2 \mathrm{H})$ \\
\hline 24 & & $2.18(\mathrm{~m}, 1 \mathrm{H})$ & 34.4 & & & & $2.27(\mathrm{~m}, 1 \mathrm{H})$ \\
\hline 25 & & $1.60(\mathrm{~m}, 1 \mathrm{H})$ & 29.4 & & & & $1.63(\mathrm{~m}, 1 \mathrm{H})$ \\
\hline 26 & & $\begin{array}{l}0.96 \\
(\mathrm{~d}, 3 \mathrm{H}, J=7.0 \mathrm{~Hz})\end{array}$ & 19.4 & & & & $0.94(\mathrm{~d}, 3 \mathrm{H}, J=9 \mathrm{~Hz})$ \\
\hline 27 & & $\begin{array}{l}0.94 \\
(\mathrm{~d}, 3 \mathrm{H}, J=7.0 \mathrm{~Hz})\end{array}$ & 20.9 & & & & $0.94(\mathrm{~d}, 3 \mathrm{H}, J=9 \mathrm{~Hz})$ \\
\hline 28 & & $1.37(\mathrm{~m}, 2 \mathrm{H})$ & 24.9 & & & & $1.36(\mathrm{~m}, 2 \mathrm{H})$ \\
\hline 29 & & $\begin{array}{l}0.87 \\
\text { (distorted t, 3H) }\end{array}$ & 14.1 & & & & $0.86(\mathrm{t}, 3 \mathrm{H}, J=6.9 \mathrm{~Hz})$ \\
\hline $1^{\prime}$ & & & & & $\begin{array}{l}2.34 \\
(\mathrm{t}, 2 \mathrm{H}, J=7.4 \mathrm{~Hz}) \\
2.16 \\
(\mathrm{br},-\mathrm{N}-\mathrm{H} \text { proton })\end{array}$ & 57.6 & \\
\hline $2^{\prime}$ & & & & & $1.63(\mathrm{~m}, 2 \mathrm{H})$ & 24.43 & \\
\hline $3^{\prime}$ & & & & & $\begin{array}{l}0.86 \\
(\mathrm{t}, 3 \mathrm{H}, J=6.28 \mathrm{~Hz})\end{array}$ & 14.13 & \\
\hline $4^{\prime}$ & & & & $\begin{array}{l}8.08 \\
(\mathrm{~d}, 1 \mathrm{H}, J=8.0 \mathrm{~Hz})\end{array}$ & & & \\
\hline $5^{\prime}$ & & & & $7.47(\mathrm{dd}, 1 \mathrm{H}, J=8.0$ and $7.2 \mathrm{~Hz})$ & & & \\
\hline $6^{\prime}$ & & & & $7.60(\mathrm{~d}, 1 \mathrm{H}, J=7.2 \mathrm{~Hz})$ & & & \\
\hline
\end{tabular}


we can assign the structure of compound $\mathbf{1}$ as ethyl 3,3-dimethyl-13-hydroxy tridecanoate, which appears to be new.

\subsubsection{Compound 2}

Compound 2 was identified as stigmasta-5, 22-dien-3 $\beta$ ol by comparing its spectral data with those published for this compound [21] (Additional file 1).

\subsubsection{Compound 3}

Compound 3 was identified as tert.butyl tridecanoate by comparing its spectral data with those reported for this compound (http://www.nmrdb.org) (Additional file 1).

\subsubsection{Compound 4}

The IR spectrum of compound 4 showed an absorption band at $3676 \mathrm{~cm}^{-1}$ indicated a hydroxyl group $(-\mathrm{OH})$ and the band at 1685 is responsible for $\mathrm{C}=\mathrm{O}$ bond. The sharp absorption band at 2940 and $2869 \mathrm{~cm}^{-1}$ were demonstrative of aliphatic $\mathrm{C}-\mathrm{H}$ stretching. The bands at 983 and $883 \mathrm{~cm}^{-1}$ were demonstrative for the steroidal nature [22]. ${ }^{1} \mathrm{H}-\mathrm{NMR}$ data (Table 1 ) of $\mathbf{4}$ showed two singlets at $\delta 0.74$ and $0.81\left(2 \times \mathrm{CH}_{3}, \mathrm{C}-18,19\right)$ of $3 \mathrm{H}$ proton intensity of each and two doublets found at $\delta 0.94$ and $0.96\left(2 \times \mathrm{CH}_{3}, \mathrm{C}-26,27\right)$ of $3 \mathrm{H}$ proton. Moreover, $3 \mathrm{H}$ distorted triplet found at $\delta 0.87\left(1 \times \mathrm{CH}_{3}, \mathrm{C}-29\right)$ is typical steroidal signal [22]. The distorted triplet for single proton at $\delta 4.15$ is suggested for an oxymethine proton flanked by one methylene groups of cyclohexane ring system of a steroidal compound. The oxymethine proton may be attached to $\mathrm{C}-1, \mathrm{C}-2, \mathrm{C}-6$, or $\mathrm{C}-12$. If the oxymethine proton is attached to $\mathrm{C}-1, \mathrm{C}-2$, or $\mathrm{C}-12$, it will find a triplet, but oxymethine proton showed a broad multiplet at $\delta 4.15$ due to its $\beta$-axial orientation [23]. So, the oxymethine proton is at C-6. The spectrum displayed signals at $\delta 4.73$ and $4.65(1 \mathrm{H}$, each, s, br) attributable to an exomethylene protons [24]. The presence of the double bonds at C-20 in this structure received support from ${ }^{13} \mathrm{C}$-NMR data (Table 1 ) at $\delta 150.79$ for C-20 and $\delta 109.2$ for $\mathrm{C}-21$. The presence of a keto group (C3 ) and a hydroxyl group (C-6) is also confirmed by the ${ }^{13} \mathrm{C}-\mathrm{NMR}$ at $\delta 180.3$ for $\mathrm{C}-3$ and $\delta 79.1$ for C-6. Moreover, it responded to the Salkowsky and LiebermannBurchard [25] color tests to exhibit its steroidal nature. On the basis of spectral data, we can assign the structure of compound $\mathbf{4}$ as 6 - $\alpha$-hydroxy stigmast-20(21)-en-3one. The structure of $\mathbf{4}$ was confirmed on the basis of the comparison of their data with lupeol [24] and $12 \alpha-$ Hydroxystigmast-4-en-3-one [23].

\subsubsection{Compound 5}

The IR spectrum of compound $\mathbf{5}$ was assigned for the presence of hydroxyl group $(-\mathrm{OH})$ at $3425 \mathrm{~cm}^{-1}$. The band at $1506 \mathrm{~cm}^{-1}$ is responsible for double bond stretching of aromatic carbon, whereas the band at 1660 $\mathrm{cm}^{-1}$ indicated the aliphatic $\mathrm{C}=\mathrm{C}$ stretching. From the ${ }^{1} \mathrm{H}$-NMR data (Table 1), a doublet of doublet at $\delta 7.47$ $\left(J=8.0\right.$ and $\left.7.2 \mathrm{~Hz}, \mathrm{H}-5^{\prime}\right)$ and two doublets at $\delta 7.60(J=$ $\left.7.2 \mathrm{~Hz}, \mathrm{H}-6^{\prime}\right)$ and $8.08\left(\mathrm{~J}=8.0 \mathrm{~Hz}, \mathrm{H}-4^{\prime}\right)$ indicated the presence of a trisubstituted benzene ring. A distorted triplet at $\delta 5.42$ indicated the presence of a single olefinic proton at $\mathrm{H}-17$. The absorption band at $\delta 3.87(\mathrm{~m})$ indicated a $\mathrm{H}-2$ proton which is attached to the oxygen $(-\mathrm{O}-)$ atom of hydroxyl group. Whereas $=\mathrm{C}-\mathrm{CH}_{3}$ group is shown by the singlet at $\delta 2.03$ of $3 \mathrm{H}$. It also showed $3 \mathrm{H}$ intensity at $\delta 0.86(\mathrm{~d})$. A distorted triplet found at $\delta$ 2.35 of $\mathrm{H}-16$ and multiplets of $26 \mathrm{H}\left(13 \times \mathrm{CH}_{2}\right)$ are confirmed at $\delta 1.24-1.53$. The ESI-MS (positive ion) $\mathrm{m} / \mathrm{z}$ 413.2 is corresponding to $\left(\mathrm{M}^{+}+\mathrm{Na}\right)$ and GC-MS m/z 391 for $\left(\mathrm{M}^{+}+\mathrm{H}\right)$ which will be at 390 . Thus, the molecular formula of compound 5 is $\mathrm{C}_{25} \mathrm{H}_{42} \mathrm{O}_{3}$. On the basis of IR, ${ }^{1} \mathrm{H}-\mathrm{NMR}$, and mass spectral data, we have assigned the structure of compound $\mathbf{5}$ as 18-(2', 3'-dihydroxyphenyl)nonadec-17-en-2-ol.

\subsubsection{Compound 6}

Strong IR absorption band at $1701 \mathrm{~cm}^{-1}$ clearly indicated the presence of a keto (-CO) group. On the other hand, absorption band at $3411 \mathrm{~cm}^{-1}$ indicated $\mathrm{N}-\mathrm{H}$ stretching and $\mathrm{C}-\mathrm{N}$ bond confirmed at 1652 and $1635 \mathrm{~cm}^{-1}$. A broad peak at $\delta 2.16$ in the ${ }^{1} \mathrm{H}$-NMR data (Table 1) assigned for $\mathrm{N}-\mathrm{H}$ proton. The ${ }^{13} \mathrm{C}$-NMR also showed the peak at $\delta$ 57.6 indicated that amino-substituted carbon is there. The $\mathrm{C}-1$ is flanked by a keto group as well as by $\mathrm{NH}$ group is indicated by the ${ }^{1} \mathrm{H}-\mathrm{NMR}$ value at $\delta 4.04(\mathrm{~s}, \mathrm{br})$ and ${ }^{13} \mathrm{C}$ NMR value at $\delta$ 67.5. The n-propyl substituent of $\mathrm{NH}$ is indicated by the peaks at $\delta 0.8(\mathrm{t}, 3 \mathrm{H}), 1.63(\mathrm{~m}, 2 \mathrm{H})$, and $2.34(\mathrm{t}, 2 \mathrm{H})$. The other broad absorptions at $\delta 1.24$ corresponding to $34 \mathrm{H}$ indicated the 17 -methylene groups in the long chain. The ${ }^{13} \mathrm{C}-\mathrm{NMR}$ data (Table 1) assumed 24 carbon atoms and therefore the molecular formula can be calculated as $\mathrm{C}_{24} \mathrm{H}_{49} \mathrm{NO}$. On the basis of spectral data, the 6 assigned for 1-(N-propyl amino)-2-henecosanone.

\subsubsection{Compound 7}

${ }^{1} \mathrm{H}$-NMR data (Table 1 ) of compound 7 showed a typical steroidal type compound [22]. Signals which observed two singlets at $\delta 0.74$ and $0.81\left(2 \times \mathrm{CH}_{3}, \mathrm{C}-18,19\right.$ of $3 \mathrm{H}$ proton of each), two doublets at $\delta 0.94\left(2 \times \mathrm{CH}_{3}, \mathrm{C}-26\right.$, $27)$, and a $3 \mathrm{H}$-distorted triplet at $\delta 0.86\left(1 \times \mathrm{CH}_{3}, \mathrm{C}-29\right)$. On the other hand, the distorted triplet at $\delta 5.35$ is suggestive of an alkene proton of cyclohexane ring system of a steroidal compound [23]. The spectrum attributable to an exomethylene protons at $\delta 4.72$ and $4.59(1 \mathrm{H}$, each, br.s) [24]. The ${ }^{1} \mathrm{H}-\mathrm{NMR}$ for $\mathrm{H}-2$ at $\delta 3.17$ and $\mathrm{H}-4$ at $\delta 3.65$ (s) indicated that the keto group is present in $\mathrm{C}-3$ position. We could not do any ${ }^{13} \mathrm{C}$-NMR and mass spectra due to the isolation of a very small amount of 7 . But the ${ }^{1} \mathrm{H}$-NMR spectra is almost similar to that for 
compound 4. Moreover, it responded to the Salkowsky and Liebermann-Burchard color tests [25] of steroidal compounds. Therefore, ${ }^{1} \mathrm{H}-\mathrm{NMR}$ spectral data of compound 7 is suggested as stigmast-5(6), 20(21)-diene-3one by comparison with $\mathbf{4}$ which molecular formula is $\mathrm{C}_{29} \mathrm{H}_{46} \mathrm{O}$. The structure of 7 is attained only by the removal of water from 4 , that results in a $\mathrm{C}=\mathrm{C}$ double bond at $\mathrm{C}-5$ and $\mathrm{C}-6$.

\section{Conclusions}

Adenanthera pavonina Linn. has been reported for its various pharmacological activities in the field of traditional medicines. Present investigation has unfolded its seven compounds from the bark extract especially dichloromethane and ethyl acetate extract for the first time. The compounds were isolated through chromatographic methods and their structures were established by extensive spectroscopic techniques, particularly NMR. This investigation may open up future research in the field of synthetic chemistry to synthesis new series of compounds with immense medicinal importance.

\section{Supplementary information}

Supplementary information accompanies this paper at https://doi.org/10. 1186/s43088-019-0013-0.

Additional file 1: Figure S1. ${ }^{1} \mathrm{H}-\mathrm{NMR}$ spectrum of the compound 1. Figure S2. ESI-MS (Negative ion) spectrum of the compound 1. Figure S3. IR spectrum of the compound 2. Figure S4. ${ }^{1} \mathrm{H}-\mathrm{NMR}$ spectrum of the compound 2. Figure S5. ${ }^{13} \mathrm{C}-\mathrm{NMR}$ spectrum of the compound 2. Figure S6. ESIMS spectrum of the compound 2. Figure S7. IR spectrum of the compound 3. Figure S8. ${ }^{1} \mathrm{H}-\mathrm{NMR}$ spectrum of the compound 3. Figure S9. ${ }^{13} \mathrm{C}-\mathrm{NMR}$ spectrum of the compound 3. Figure S10. IR spectrum of the compound 4. Figure S11. ${ }^{1} \mathrm{H}-\mathrm{NMR}$ spectrum of the compound 4. Figure S12. ${ }^{13} \mathrm{C}-\mathrm{NMR}$ spectrum of the compound 4. Figure S13. IR spectrum of the compound 5. Figure S14. ${ }^{1} \mathrm{H}-\mathrm{NMR}$ spectrum of the compound 5. Figure S15. ESI-MS (positive ion) spectrum of the compound 5. Figure S16. GC-MS spectrum of the compound 5. Figure S17. IR spectrum of the compound 6. Figure S18. ${ }^{1} \mathrm{H}-\mathrm{NMR}$ spectrum of the compound 6. Figure S19. ${ }^{13} \mathrm{C}$-NMR spectrum of the compound 6. Figure S20. ${ }^{1} \mathrm{H}-\mathrm{NMR}$ spectrum of the compound 7. Table S1. ${ }^{1} \mathrm{H}-\mathrm{NMR}$ of compound 2 and Comparison of ${ }^{13} \mathrm{C}-\mathrm{NMR}$ data of compound 2 with those of the published data [19]. Table $\mathbf{S 2} .{ }^{13} \mathrm{C}$ NMR and $1 \mathrm{H}-\mathrm{NMR}$ data of compound 3 .

\section{Abbreviations}

APT: Attached proton test; br.s: Broad singlet; CC: Column chromatography; DCM: Dichloromethane; DEPT: Distortionless enhancement by polarization transfer; HPLC: High-performance liquid chromatography; IR: Infrared spectroscopy; LC-ESI-MS: Liquid chromatography-electron spray ionizationMass spectroscopy; NMR: Nuclear magnetic resonance; PTLC: Preparative thin layer chromatography; VLC: Vacuum liquid chromatography

\section{Acknowledgements}

The authors express thanks to BCSIR laboratory, Dhaka, Bangladesh, and Leibniz institute of Plant Biochemistry, Halle (Saale), Germany, for the instrumental facilities offered in recording the spectra. Arzumand Ara is grateful to the ministry of education, Government of the Republic of Bangladesh and University Grants Commission (UGC) for awarding a fellowship.

\section{Authors' contributions}

AR designed and carried out the chromatographic separation and isolated the compounds. MMS participated in the characterization of compounds and drafted the manuscript. MAH supervised the experiment. MA participated in the elucidation of structures of compounds and supervised the study. $\mathrm{CMH}$ elucidated the structure of the isolated compounds and checked themanuscript. All authors read and approved the final manuscript.

\section{Funding}

There has been no funding for this study.

Availability of data and materials

Not applicable

Ethics approval and consent to participate

Not applicable

Consent for publication

Not applicable

\section{Competing interests}

The authors declare that they have no competing interests.

\section{Author details}

${ }^{1}$ Department of Chemistry, Jahangirnagar University, Savar, Dhaka 1342, Bangladesh. ${ }^{2}$ Department of Pharmaceutical Chemistry, Faculty of Pharmacy, University of Dhaka, Dhaka 1000, Bangladesh.

Received: 26 July 2019 Accepted: 25 September 2019

Published online: 17 December 2019

\section{References}

1. Kirtikar KR, Basu BD (1981) Indian medicinal plants, 2nd edn. International Book Distributors, India, p 1710

2. Ghani A (2003) Medicinal plants of Bangladesh (chemical constituents and uses), 2nd edn. Asiatic Society of Bangladesh, Dhaka, pp 331-332

3. Anonymous (1985) The wealth of India, a dictionary of Indian raw materials and industrial products, vol IA. CSIR, New Delhi

4. Burkil HM (1994) The useful plants of West Tropical Africa, vol 2. Royal Botanical Gardens, Kew

5. Watt JM, Breyer-Brandwijk MG (1962) The medicinal and poisonous plants of Southern and Eastern Africa, 2nd edn. E and S Livingstone Ltd, London

6. Adedapo ADA, Osude YO, Adedapo AA, Moody JO, Adeagbo AS, Olajide OA, Makinde JM (2009) Blood pressure lowering effect of Adenanthera pavonina seed extract on normotensive rats. Rec Nat Prod 3:82-89

7. Rodrigo SK, Jayasingha ULB, Bandara BMR (2007) Antifungal, antioxidant and cytotoxic activity of Acronychia pedunculata and Adenanthera pavonina, Proceeding of the Vol12(1). Peradeniya University Research Sessions, Sri Lanka, p 94

8. Jayasinghe PKIDE, Bandara BMR, Ekanayaka EWMA, Thevanesam V (2006) Screening for antimicrobial activity of Acronychia pedunculata (Ankenda) and Adenanthera pavonina (Madatiya) against bacteria causing skin and wound infections in humans, Vol 11. Proceedings of the Peradeniya University Research Sessions, Sri Lanka, p 105

9. Olajide OA, Echianu CA, Adedapo ADA, Makinde JM (2004) Antiinflammatory studies on Adenanthera pavonina seed extract. Inflammopharmacol 12(2):196-202

10. Mayuren C, llavarasan R (2009) Anti-inflamatory activity of ethanolic leaf extracts from Adenantheran pavonina $(\mathrm{L})$ in rat. Pharmacognosy 1(2):125128

11. Ara A, Arifuzzaman M, Ghosh CK, Hashem MA, Ahmad MU, Bachar SC, Nahar L, Sarker SD (2010a) Anti-inflammatory activity of Adenanthera pavonina L., Fabaceae, in experimental animals. Rev Bras de Farmacog 20(6): 929-932

12. Ara A, Saleh-e-In MM, Ahmed NU, Ahmed MU, Hashem MA, Bachar SC (2010b) Phytochemical screening, analgesic, antimicrobial and antioxidant activities of bark extracts of Adenanthera pavonina L. (Fabaceae). Adv Nat Appl Sci 4(3): 352-360 
13. Ara A, Saleh-e-In MM, Ahmed NU, Hashem MA, Bachar SC (2013) Antidiarrheal, acute toxicity activity of methanolic bark extract and elemental composition of Adenanthera pavonina L. (Fabaceae). Turk J Pharm Sci 10(2): 263-272

14. Yadev N, Misra G, Nigam SK (1976) Triterpenoids of Adenanthera pavonina bark. Planta Med 29(2):176-178

15. Yeoh HH, Wee YC, Watson L (1984) Systematic variation of leaf amino acid compositions of leguminous plants. Phytochem 23(10):2227-2229

16. Chandra SM, Saxena VH (1982) Triterpenoids of Adenanthera pavonina root. Int J Crude Drug Res 20:165-167

17. Mesbah UA, Rahman MA, Tabassum R, Nahar K (2002) Chemical constituents of the leaves of Adenanthera pavonina L. J Bangladesh Chem Soc 15(2):194-199

18. Shaiq AM, Ahmed F, Azhar I, Pervez MK (2005) Pavinin: a new five membered lactone from Adenanthera pavonina L. (Mimoaceae). Nat Prod Res 19(1):37-40

19. Enuo Y, Shishan PY (2007) Studies on chemical constituents from stems and leaves of Adenanthera pavonina L. Zhongguo Zhongyao Zazhi 32(20):21352138

20. Sudhakar PK, Pattabiraman TN, Thillaisthanan N (1980) Natural plant enzyme inhibitors. Isolation and characterization of a trypsin/chymotrypsin inhibitor from Indian red wood (Adenanthera pavonina) seeds. J Sci Food Agric 31(10):967-980

21. Xie DL, Wang HY, Li G (2000) Isolation and production of artemisinin and stigmasterol in hairy root cultures of Artemisia annua. Plant Cell Tissue Org Cult 63:161-166

22. Finar IL (1975) Organic chemistry, vol 2, 5th edn. ELBS, Longman publishers, Singapore, p 696

23. Chowdhury R, Rashid RB, Sohrab MH, Hasan CM (2003) 12aHydroxystigmast-4-en-3-one: a new bioactive steroid from Toona ciliate (Meliaceae). Pharmazie 58:272-273

24. Nazma PM, Mohammad SR, Mohammad SI, Mohammad AR (2009) Chemical and biological investigations of Dillenia indica Linn. Bangladesh J Pharrmacol 4:122-125

25. Sofowara A (1993) Medicinal plants and traditional medicine in Africa. Spectrum Books Ltd, Ibadan, p 289.

\section{Publisher's Note}

Springer Nature remains neutral with regard to jurisdictional claims in published maps and institutional affiliations.

\section{Submit your manuscript to a SpringerOpen ${ }^{\circ}$ journal and benefit from:}

- Convenient online submission

- Rigorous peer review

- Open access: articles freely available online

High visibility within the field

- Retaining the copyright to your article

Submit your next manuscript at $\boldsymbol{\nabla}$ springeropen.com 